12 Gueyffier F, Boutitie F, Boissel J-P, Pocock S, Coope J, Cutler J, et al. Effect of antihypertensive drug treatment on cardiovascular outcomes in women and men. Ann Intern Med 1997;126:761-7.

13 Coope J, Warrender TS. Randomised trial of treatment of hypertension in elderly patients in primary care. BMJ 1986;293:1145-51.

14 Amery A, Birkenhäger W, Brixko P, Bulpitt C, Clément D, Deruyttere M, et al. Mortality and morbidity results from the European working party on high blood pressure in the elderly trial. Lancet 1985;i:1349-54.

15 Hypertension Detection and Follow-up Program Cooperative Group Five-year findings of the hypertension detection and follow-up program I. Reduction in mortality of persons with high blood pressure, including mild hypertension. JAMA 1979;242:2562-71.

16 Medical Research Council Working Party. MRC trial of treatment of mild hypertension: principal results. BMJ 1985;291:97-104.

17 MRC Working Party. Medical Research Council trial of treatment of hypertension in older adults: principal results. BMJ 1992;304:405-12.

18 Multiple Risk Factor Intervention Trial Research Group. Multiple risk factor intervention trial: risk factor changes and mortality results. JAMA 1982;248:1465-77.

19 SHEP Cooperative Research Group. Prevention of stroke by antihypertensive drug treatment in older persons with isolated systolic hypertension: final results of the systolic hypertension in the elderly program (SHEP). JAMA 1991;265:3255-64.

20 Dalhöf B, Lindholm L, Hansson L, Shersten B, Ekbom T, Wester PO. Morbidity and mortality in the Swedish trial in old patients with hypertension (STOP-Hypertension). Lancet 1991;338:1281-5.

21 Kannel WB. Historic perspectives on the relative contributions of diastolic and systolic blood pressure elevation to cardiovascular risk profile. $A m$ Heart J 1999;138:205-10.

22 Shaper AG, Phillips AN, Pocock SJ, Walker M, Macfarlane PW. Risk factors for stroke in middle aged British men. BMJ 1991;302:1111-5.

23 World Health Organization. World health statistics annual 1998. Geneva: WHO, 1998

24 Wannamethee SG, Shaper AG, Perry IJ. Serum creatinine concentration and risk of cardiovascular disease. Stroke 1997;28:557-63.

25 Wannamethee SG, Shaper AG, Whincup PH, Walker M. Adult height, stroke and coronary heart disease. Am J Epidemiol 1998;148:1069-76.

26 Kannel WB, McGee DL. Diabetes and cardiovascular disease: the Framingham study. JAMA 1979;241:2035-8.

(Accepted 10 April 2001)

\title{
Reduced osmolarity oral rehydration solution for treating dehydration due to diarrhoea in children: systematic review
}

\author{
Seokyung Hahn, YaeJean Kim, Paul Garner
}

\begin{abstract}
Objectives To compare reduced osmolarity oral rehydration solution with standard World Health Organization oral rehydration solution in children with acute diarrhoea.

Design Systematic review of randomised controlled trials.

Studies 15 randomised controlled trials including 2397 randomised patients.
\end{abstract}

Outcomes The primary outcome was unscheduled intravenous infusion; secondary outcomes were stool output, vomiting, and hyponatraemia.

Results In a meta-analysis of nine trials for the primary outcome, reduced osmolarity rehydration solution was associated with fewer unscheduled intravenous infusions compared with standard WHO rehydration solution (odds ratio $0.61,95 \%$ confidence interval 0.47 to 0.81 ). Three trials reported that no patients required unscheduled intravenous infusion. Trials reporting secondary outcomes suggested that in the reduced osmolarity rehydration solution group, stool output was lower (standardised mean difference in the log scale -0.214 (95\% confidence interval -0.305 to $-0.123 ; 13$ trials) and vomiting was less frequent (odds ratio 0.71, 0.55 to 0.92 ; six trials). Six trials sought presence of hyponatraemia, with events in three studies, but no significant difference between the two arms.

Conclusion In children admitted to hospital with dehydration associated with diarrhoea, reduced osmolarity rehydration solution is associated with reduced need for unscheduled intravenous infusions, lower stool volume, and less vomiting compared with standard WHO rehydration solution.

\section{Introduction}

Diarrhoea remains a leading cause of childhood death in developing countries. The main complication is dehy- dration, which until the early 1960s was treated with intravenous infusion. Solutions of oral rehydration salts are now the main treatment and are particularly useful when intravenous fluids are in short supply, health services are basic, and there is a shortage of skilled staff.' The combination of salt and sugar probably enhances absorption of fluid because sodium and glucose transport in the small intestine are coupled; glucose promotes absorption of both sodium ions and water. Oral rehydration salts have proved both safe and effective worldwide in hospital settings and are now widely used in the home to prevent dehydration. ${ }^{34}$

For more than two decades, the World Health Organization has recommended a standard formulation of glucose based oral rehydration solution with 90 $\mathrm{mmol} / \mathrm{l}$ of sodium and $111 \mathrm{mmol} / \mathrm{l}$ of glucose and a total osmolarity of $311 \mathrm{mmol} / \mathrm{l}$. It remains unclear however, whether this is the optimum sodium concentration. Some studies have found patients with blood sodium concentrations above the normal level of 150 $\mathrm{mmol} / \mathrm{l}{ }^{5}$ Laboratory work suggests that lower concentrations of sodium and glucose enhance solute induced water absorption. ${ }^{67}$

We conducted a systematic review of all relevant randomised controlled trials comparing the effects of reduced osmolarity and standard WHO oral rehydration solutions. We confined the review to children, as they are most vulnerable to dehydration and electrolyte imbalance from diarrhoea.

\section{Methods}

\section{Study inclusion and characteristics}

We included only randomised controlled trials, defined as a trial in which the subjects were assigned prospectively to one of two or more interventions by random allocation. This excludes quasirandomised designs. Patients included were children with acute diarrhoea for less than 5 days who were treated either by reduced

\section{Editorial by Fuchs}

Medical and Pharmaceutical Statistics Research Unit, University of Reading, Reading RG6 6FN

Seokyung Hahn research fellow

Department of Paediatrics, Seoul

National University Children's Hospital, Seoul 110-774,

South Korea

YaeJean Kim paediatrician

Effective Health Care Alliance Programme, International Health Division, Liverpool School of Tropical Medicine, Liverpool L3 5QA Paul Garner professor

Correspondence to: S Hahn,

Department of Health Sciences and Clinical Evaluation,

University of York, York YO10 5DD s.hahn@rdg.ac.uk

BMJ 2001;323:81-5

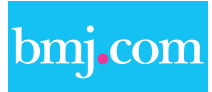

Tables giving further details of the included trials are available on the BMJ's website 
osmolarity oral rehydration solution (total osmolarity $\leqslant 250 \mathrm{mmol} / 1$ with reduced sodium) or by standard WHO oral rehydration solution (sodium $90 \mathrm{mmol} / \mathrm{l}$, glucose $111 \mathrm{mmol} / \mathrm{l}$, total osmolarity $311 \mathrm{mmol} / \mathrm{l}$ ).

The primary outcome was specified as the need for unscheduled intravenous infusion during the course of treatment. This was defined as clinical requirement for intravenous infusion after oral rehydration had been started. We chose unscheduled intravenous infusion as the primary outcome because it is a pragmatic outcome that is relevant to health providers and represents failed oral treatment. Other outcomes were stool output, vomiting during rehydration, and presence of hyponatraemia (serum sodium concentration $<130 \mathrm{mmol} / \mathrm{l}$ ) during follow up.

\section{Search strategy}

We searched the following databases for published clinical trials: Medline (1966 to June 2001); Embase (1988 to May 2001); Cochrane controlled trials register in the Cochrane Library (Issue 2, 2001); and Current Contents (June 2001). We used child, diarrhoea, fluid therapy, oral rehydration, osmolar, and rehydration solutions as search terms. We also checked the citations of existing reviews and trial reports. For unpublished data and ongoing trials, we contacted current researchers and key agencies, including the WHO, the Centers for Disease Control, Atlanta, and the International Centre for Diarrhoeal Disease Research, Bangladesh.

\section{Data extraction and synthesis}

We used the standard methods of the Cochrane infectious diseases group to prepare the protocol, apply inclusion criteria, assess quality, and extract data. We assessed quality by adequacy of concealment of allocation, generation of allocation sequence, blinding, and follow up of patients. The first two authors independently extracted data on relevant outcome measures using a standardised data abstraction form, and any disagreements were resolved by discussion.

We used the Mantel-Haenszel odds ratio for binary outcomes. The odds ratios were not estimated when neither intervention group found any event. We used the standardised mean difference for continuous outcomes. We combined studies using a fixed effect method based on a weighted average of the results with weights proportional to the inverse of the variance. ${ }^{8}$

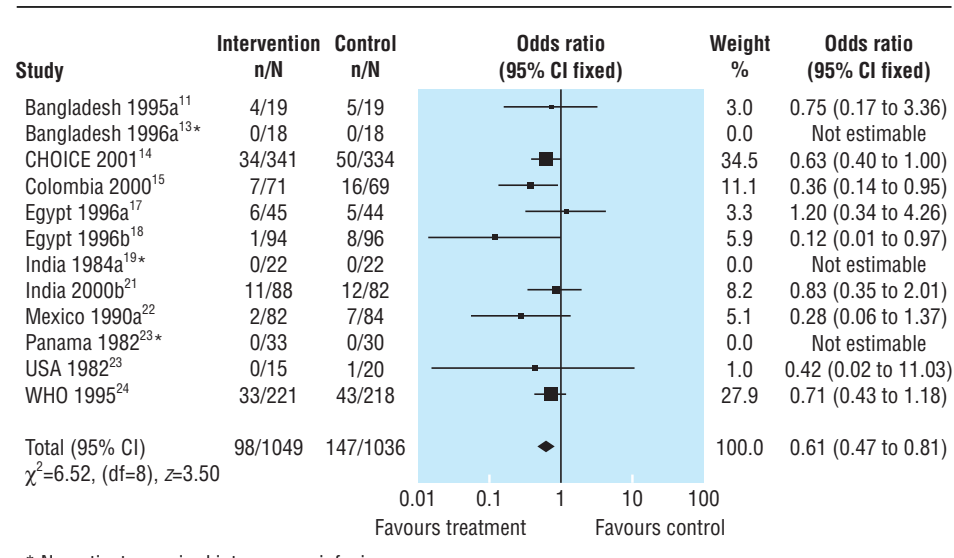

* No patients required intravenous infusion

Fig 1 Meta-analysis of unscheduled intravenous infusion among children randomised to reduced osmolarity and standard WHO rehydration solutions
For all estimates, we calculated 95\% confidence intervals. We tested statistical heterogeneity using $\chi^{2}$ tests, with a $\mathrm{P}$ value $\leqslant 0.1$ indicating significance. We had prespecified potential sources of heterogeneity for analysis. ${ }^{9}$ We examined publication bias using a funnel plot and a regression approach to assess asymmetry of the plot. ${ }^{10}$ We also did a sensitivity analysis to assess the effect of adequacy of concealment of allocation.

\section{Results}

\section{Profile of studies}

Of 41 identified studies, six were not randomised trials, eight had not used glucose based reduced osmolarity rehydration solution, six had not used standard WHO rehydration solution, two were in adults, and two did not report on any of the relevant outcomes. Seventeen studies in 16 published reports met the inclusion criteria. ${ }^{11-26}$ One paper reported on two trials, ${ }^{24}$ one in the United States and one in Panama, and we present these as separate studies. We contacted the authors of three papers, ${ }^{22}{ }^{26}$ as we judged these were three reports of the same trial. As we have not received a response, we included only the paper with the largest number of patients. ${ }^{22}$

\section{Description and quality of studies}

Details of the 15 trials included in the analysis and their patient characteristics are available on the $B M J$ s website. Included studies were from Egypt (three studies), Bangladesh (three), Mexico (one), Colombia (one), India (three), Panama (one), and the United States (one). Two other studies were multicentre trials; one was conducted in Brazil, India, Mexico, and Peru and the other in Bangladesh, Brazil, India, Peru, and Vietnam.

Three trials included children with cholera. ${ }^{12} 1421$ Children were aged 1-36 months in all trials except one, which included children up to 5 years old. ${ }^{21}$ All children had some degree of clinical dehydration. One trial treated all children on day 1 with intravenous infusion, and those with a stool output of $80 \mathrm{ml} / \mathrm{kg} / 24 \mathrm{~h}$ were then randomised. ${ }^{11}$ Five trials included children with severe dehydration. ${ }^{14212324}$ Five trials included malnourished children. ${ }^{12}{ }^{15} 20-22$ The number of breastfed children was reported in eight trials. ${ }^{11-15} 182124$ Fully weaned children were included in one trial. ${ }^{16}$

We deviated slightly from the osmolarity definitions in our refereed protocol published in the Cochrane Library. ${ }^{9}$ We defined reduced osmolarity as $<250$ $\mathrm{mmol} / \mathrm{l}$, but some studies defined reduced osmolarity as higher than this, and we therefore extended our limit to $<270 \mathrm{mmol} / \mathrm{l}$. We also included two studies that used a WHO oral rehydration solution with a total osmolarity of $331 \mathrm{mmol} / \mathrm{l}$ rather than $311 \mathrm{mmol} / \mathrm{l}$ but with the same sodium and glucose combination. ${ }^{23}$ All but three trials used a glucose based reduced osmolarity rehydration solution; one used sucrose, ${ }^{13}$ another maltodextrin, ${ }^{17}$ and the third used L-alanine with glucose. ${ }^{11}$

Nine trials reported methods that assured adequate concealment of allocation. Seven studies stated they were double blinded, and eight did not mention blinding.

\section{Quantitative data synthesis}

Figures 1-4 show the meta-analyses for the four outcomes. Information on the primary outcome 
(unscheduled intravenous infusion) was available in 12 trials $(n=2085)$, but in three of these no patients in either group required infusion. In the meta-analysis of nine trials, the need for intravenous infusion was significantly reduced for participants who received reduced osmolarity rehydration solution compared with those receiving WHO rehydration solution (odds ratio $0.61,95 \%$ confidence interval 0.47 to 0.81 ).

Thirteen trials reported stool output during rehydration. These trials measured stool output in various ways using different units. We therefore used the standardised mean difference to analyse these data. Since the stool output in diarrhoeal disease showed a positive skewed distribution with clinical improvement, we used a log-normal approximation. The pooled standardised mean difference in the log scale is -0.214 (95\% confidence interval -0.305 to -0.123 ), which suggests that the reduced osmolarity rehydration solution resulted in significantly less stool output than the WHO solution. Data from one trial $^{19}$ were not combined with the others in the meta-analysis because this trial measured stool output for much longer than the others (see $B M$ /s website for details of results).

Six trials reported on vomiting during rehydration. The tendency was for fewer patients to vomit in the reduced osmolarity rehydration solution group (odds ratio $0.71,0.55$ to 0.92 ).

Six trials reported on hyponatraemia. In three of these six trials, no patients had hyponatraemia. The meta-analysis of the three trials in which participants developed hyponatraemia showed no significant difference between the groups (odds ratio 1.45, 0.93 to 2.26). We found no evidence of statistical heterogeneity of treatment effect for any of the four outcomes.

The funnel plot of the primary outcome showed no significant evidence of publication bias (fig 5). The regression method used to assess funnel plot asymmetry gave an intercept of -0.72 with a $P$ value of 0.29 . A sensitivity analysis restricted to studies with clear evidence of adequate concealment of allocation produced results that did not differ greatly from those of the full meta-analysis. For example, the pooled odds ratios of the seven trials for the primary outcome with adequate concealment of allocation was 0.61 ( 0.46 to $0.82)$.

\section{Discussion}

We found that reduced osmolarity rehydration solution was more effective than standard WHO rehydration solution in first line treatment of children with diarrhoea. It reduced the need for unscheduled intravenous infusion, stool output during rehydration, and the number of patients with vomiting during oral rehydration treatment. The reduced osmolarity rehydration solution did not seem to increase the risk of developing hyponatraemia compared with the standard WHO solution, although we cannot exclude this possibility.

We examined trials of oral rehydration salts in children admitted to hospital with dehydration because of diarrhoea. The trials do not provide any direct evidence for or against use of oral rehydration solutions at home to prevent dehydration; nor do they provide any direct evidence that reduced osmolarity solutions are more effective in preventing dehydration

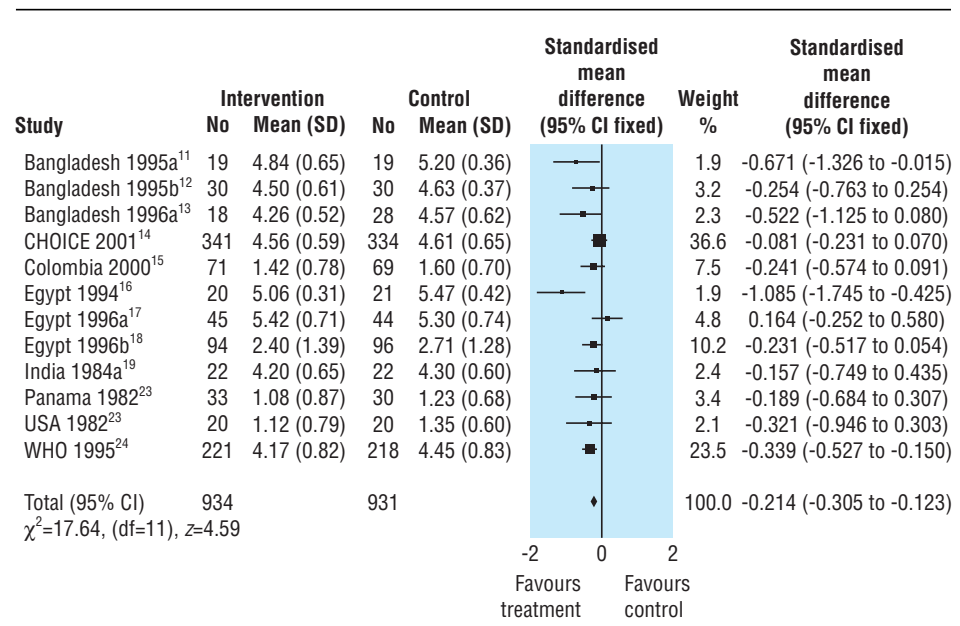

Fig 2 Meta-analysis of stool output among children randomised to reduced osmolarity and standard WHO rehydration solutions

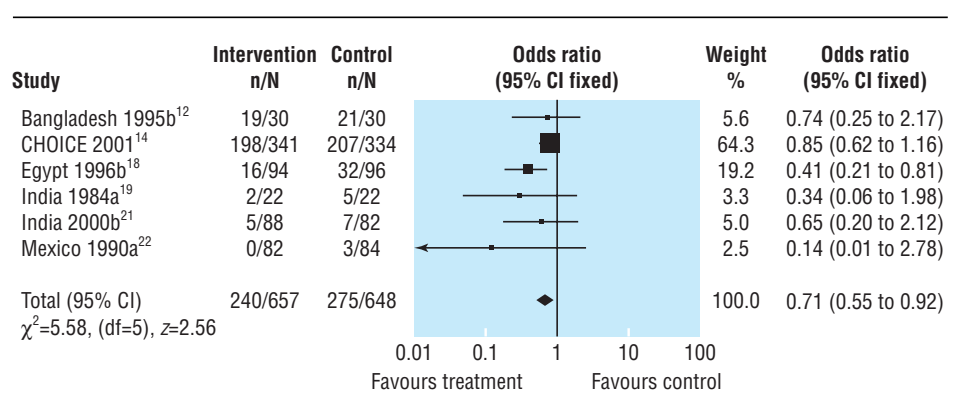

Fig 3 Meta-analysis of vomiting among children randomised to reduced osmolarity and standard WHO rehydration solutions

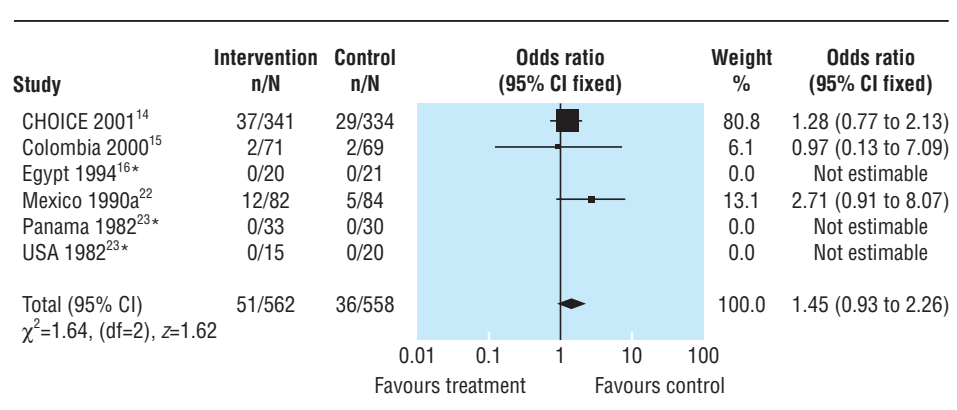

* No patients had hyponatraemia

Fig 4 Meta-analysis of hyponatraemia among children randomised to reduced osmolarity and standard WHO rehydration solutions

in the home. Oral rehydration solutions are widely used to prevent dehydration, and further research is therefore needed in this area.

\section{Choice of primary outcome}

We stand by our selection of unscheduled intravenous infusion rather than volume of diarrhoea as the primary outcome. ${ }^{9}$ Some specialists consider that volume of diarrhoea is more appropriate, probably because it reflects the animal and human perfusion experiments that provide part of the rationale for a reduced osmolarity rehydration solution. Unscheduled intravenous infusion is pragmatic; it provides a measure of failed oral rehydration and has implica- 
tions for healthcare resources. For these reasons, we preserved this as the primary outcome.

When we reviewed the studies for inclusion, most trials reported unscheduled intravenous infusion in the details of trial implementation. As this was identified as our primary outcome, we sought out these data and presented them as the primary analysis. We believe that the analysis shows a clear effect. Our approach highlights the value of paying careful attention to the protocol for a systematic review before examining the trials and illustrates how non-specialist viewpoints can help obtain practical and useful answers from a meta-analysis.

\section{Cholera}

We intended to examine treatment effects in patients with and without cholera. ${ }^{9}$ A Cochrane review of rice based rehydration compared with glucose oral rehydration solution showed that rice water was associated with lower stool volumes in cholera patients but not in diarrhoea from other causes. ${ }^{27}$ The available data were, however, insufficient. Three studies included cholera patients, ${ }^{12121}$ but separate data for cholera patients were not available. We excluded two studies in patients with cholera because they were in adults. ${ }^{28} 29$ Any recommendation for rehydration treatment for adults with cholera will need to take into account these and any other trials found through careful systematic searching.

Patients with cholera have severe loss of electrolytes. It is unclear, therefore, whether reduced osmolarity rehydration solution would be more effective than standard WHO rehydration solution in these patients. The reduced osmolarity solution could increase the risk of hyponatraemia and result in adverse clinical events

\section{Implications}

Our study suggests that reduced osmolarity rehydration solution should replace the WHO solution as the standard treatment for dehydration caused by diarrhoea. Policymakers and clinicians may, however, consider that the risk of hyponatraemia in patients with cholera outweighs the advantages of a reduced osmolarity solution. One option would be to provide standard WHO rehydration solution for people with suspected cholera or in areas where cholera is prevalent. This is likely to be a logistical problem in areas where diarrhoea is common and coexists with

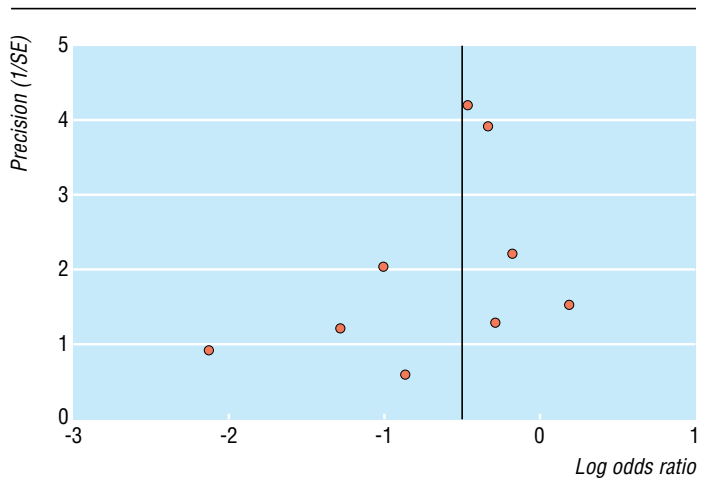

Fig 5 Funnel plot for the nine trials in which unscheduled intravenous infusion was required
What is already known on this topic

Oral rehydration solution prevents death from diarrhoea in many developing countries

\section{What this study adds}

Children receiving a reduced osmolarity rehydration solution were less likely to need intravenous infusion than those receiving WHO rehydration solution

Reduced osmolarity rehydration solution also reduced stool output and vomiting

No difference was found in rates of hyponatraemia

cholera. The single formula sachet aids implementation of this lifesaving intervention. Providing different formulations complicates distribution and may impair the effective delivery of any oral rehydration solution to children. If policymakers decide not to use reduced osmolarity solution in areas where cholera is common, they must conduct a randomised controlled trial of the two treatments in children with cholera to determine whether the decision is correct.

This review will be maintained in the Cochrane Library. We thank Dr Olivier Fontaine (WHO) for support throughout the process; and Dr Sheila Bird, Medical Research Council, Cambridge, for her comments.

Contributors: $\mathrm{SH}$ and YK wrote the protocol, extracted, analysed and interpreted the data, and drafted the paper. PG advised on inclusion criteria and outcomes for the protocol, quality assessment, and analysis, and helped write the review. PG is the guarantor.

Funding : The study is supported by WHO and the Cochrane Infectious Diseases Group is supported by the Department for International Development.

Competing interests: None declared.

1 Almroth S, Latham MC. Rational home management of diarrhoea. Lancet 1995:345:709-11.

2 Fordtran JS, Rector FC, Carter NW. The mechanisms of sodium absorption in the human small intestine. J Clin Invest 1968;47:884-900.

3 Mahalanabis M, Choudfuri AB, Bagchi NG. Oral fluid therapy of cholera among Bangladesh refugees. Johns Hopkins Med J 1973;132:197-205.

4 Grant JP. The state of the world's children 1982-1983. New York: UNICEF, 1983.

5 Finberg L. Hypernatremic (hypertonic) dehydration in infants. $N$ Engl J Med 1973:289:196-8.

6 Farthing MJ. History and rationale of oral rehydration and recent developments in formulating an optimal solution. Drugs 1988;36(suppl 4):8090.

7 Hunt JB, Elliotte EJ, Fairclough PD, Clark ML, Farthing MJ. Water and solute absorption from hypotonic glucose-electrolyte solutions in human jejunum. Gut 1992;33:479-83.

8 Sutton AJ, Abrams KR, Jones DR, Sheldon TA, Song F. Fixed effects methods for combining study estimates. In: Methods for meta-analysis in medical research. Chichester: Wiley, 2000.

9 Kim Y, Hahn S, Garner P. Reduced osmolarity oral rehydration solution for treating diarrhoea-associated dehydration in children (protocol for a Cochrane review). In: Cochrane Collaboration. Cochrane Library. Issue 4. Oxford: Update Software, 2000.

10 Egger M, Davey Smith G, Schneider M, Minder C. Bias in meta-analyses detected by a simple graphical test. BMJ 1997;315:629-34.

11 Sarker SA, Majid N, Mahalanabis D. Alanine- and glucose-based hypo-osmolar oral rehydration solution in infants with persistent diarrhoea: a controlled trial. Acta Paediatr 1995;84:775-80.

12 Mahalanabis D, Faruque ASG, Hoque SS, Faruque SM. Hypotonic oral rehydration solution in acute diarrhoea: a controlled clinical trial. Acta Paediatr 1995:84:289-93.

13 Faruque ASG, Mahalanabis D, Hamadani J, Hoque SS. Hypo-osmolar sucrose oral rehydration solution in acute diarrhoea: a pilot study. Acta Paediatr 1996;85:1247-8.

14 CHOICE study group. Multicenter randomized double blind clinical trial to evaluate the efficacy and safety of a reduced osmolarity oral rehydration salts solution in children with acute watery diarrhoea. Pediatrics 2000;107:613-8.

15 Bernal C, Velaquez C, Garcia G, Uribe G, Palacio C. Oral hydration with a low osmolarity solution in children dehydrated by diarrheic diseases. A controlled clinical study. Saludarte 2000;1:6-23. 
16 El-Mougi M, El-Akkad N, Hendawi A, Hassan M, Amer A, Fontaine O, Pierce NF. Is a low-osmolarity ORS solution more efficacious than standard WHO ORS solution? J Pediatr Gastroenterol Nutr 1994;19(1):83-6.

17 El-Mougi M, Hendawi A, Koura H, Hegazi E, Fontaine O, Pierce NF. Efficacy of standard glucose-based and reduced osmolarity maltodextrinbased oral rehydration solution: effect of sugar malabsorption. Bull World Health Organ 1996;74(5):471-7.

18 Santosham M, Fayad I, Zikri MA, Hussein A, Amponsah A, Duggan C, et al. A double-blind clinical trial comparing World Health Organization oral rehydration solution with a reduced osmolarity solution containing equal amounts of sodium and glucose. J Pediatr 1996;128(1):45-51.

19 Bhargava SK, Sachdev HPS, Das Gupta B, Daral TS, Singh HP, Mohan M. Oral rehydration of neonates and young infants with dehydration diarrhea: comparison of low and standard sodium content in oral rehydration solutions. J Pediatr Gastroenterol Nutr 1984:3(4):500-5.

20 Dutta P, Mitra U, Dutta S, Manna B, Chatterjee MK, De A, et al Hypo-osmolar oral rehydration salts solution in dehydrating persistent diarrhoea in children: double-blind, randomized, controlled clinical trial. Acta Paediatr 2000;89:411-6.

21 Alam S, Afzal K, Maheshwari M, Shukla I. Controlled trial of hypo-osmolar versus World Health Organization oral rehydration solution. Indian Pediatr 2000;37:952-9.

22 Moreno-Sanchez H, Velasques-Jones L, Becerra FC, Faure A, Maulen I, de Leon $\mathrm{M}$, et al. A comparative study on two oral rehydration solutions (ORS) containing 60 or $90 \mathrm{mmol} / \mathrm{L}$ of sodium and of different osmolalities. Bol Med Hosp Infant Mex 1990;47(9):630-5.
23 Santosham M, Daum RS, Dillman L, Rodriguez JL, Luque S, Russel R, et al. Oral rehydration therapy of infantile diarrhea. A controlled study of well-nourished children hospitalized in the United States and Panama. New Engl J Med 1982;306(18):1070-6.

24 World Health Organization. International study group on reducedosmolarity ORS solutions. Multicentre evaluation of reduced-osmolarity oral rehydration salts solution. Lancet 1995;345:282-5.

25 Martinez-Pantaleon O, Faure-Vilchis A, Gomez-Najera RI, HernandezLopez M, Velasquez-Jones L. Comparative study of oral rehydration solutions containing either 90 or 60 millimoles of sodium per liter. Bol Med Hosp Infant Mex 1998;45(12):817-22.

26 Velasquez-Jones L, Becerra FC, Faure A, de Leon M, Moreno H, Maulen I, et al. Clinical experience in Mexico with a new oral rehydration solution with lower osmolality. Clin Ther 1990;2(suppl A):95-103.

27 Fontaine $\mathrm{O}$, Gore S, Pierce NF. Rice-based oral rehydration solution for treating diarrhoea. Cochrane Database Syst Rev 2000;(2):CD001264.

28 Faruque ASG, Mahalanabis D, Hamadani JD, Zetterstrom R. Reduced osmolarity oral rehydration salt in cholera. Scand J Infect Dis 1996;28:8790

29 Alam NH, Majumder RN, Fuchs GJ, CHOICE Study Group. Efficacy and safety of oral rehydration solution with reduced osmolarity in adults with cholera: a randomised double-blinded clinical trial. Lancet 1999;354:296-9

(Accepted 27 June 2001)

\section{Drug points}

\section{Neonatal convulsions after withdrawal of baclofen}

B D M Ratnayaka, H Dhaliwal, S Watkin, Department of Neonatal Medicine, City Hospital, Nottingham NG5 1PB

Baclofen is a skeletal muscle relaxant used for the relief of chronic severe spasticity resulting from disorders such as multiple sclerosis or traumatic injury to the spinal cord. We report convulsions in a 7 day old girl who had been exposed to baclofen during intrauterine life.

A paraplegic mother had been taking baclofen $20 \mathrm{mg}$ four times daily (Lioresal, Novartis, Surrey), oxybutanin $3 \mathrm{mg}$ three times daily, and trimethoprim $100 \mathrm{mg}$ daily, which she continued throughout her pregnancy. The pregnancy was uneventful, but the baby was delivered by ventouse extraction owing to fetal tachycardia. The Apgar score was 10 at one and five minutes (cord pH: arterial 7.33, venous 7.3)

Seven days later the baby was admitted with generalised convulsions. In retrospect the mother had noticed abnormal movements from the second day after birth. Investigations included a full septic screen for bacteriology and virology; a full blood count; serum electrolytes; liver function tests; a metabolic screen of blood, urine, and cerebrospinal fluid; urine for toxicology; and cranial ultrasonography. All gave negative results. The convulsions did not respond to phenobarbitone, phenytoin, clonazepam, lignocaine, or pyridoxine, which were tried according to our hospital's guidelines for the management of neonatal seizures. The baby received broad spectrum antibiotics until the cultures gave negative results. Electroencephalography on day 11 showed prolonged episodes of epileptic activity.

We thought that the convulsions could be due to withdrawal of baclofen. Baclofen, $1 \mathrm{mg} / \mathrm{kg}$ daily in four divided doses, was started. Thirty minutes after the first dose the convulsions stopped. Baclofen was withdrawn slowly over the next two weeks. Magnetic resonance imaging of the brain on day 17 suggested a short hypoxic ischaemic insult during the perinatal period.

Because the baby was in good condition at birth and because the convulsions were controlled within 30 minutes of starting baclofen, we concluded that the convulsions had been caused by its withdrawal. The change shown by the magnetic resonance image may have been secondary to the convulsions.

In adults the half life of baclofen is 2-6 hours (mean 3.5 hours). A previous report of baclofen overdose showed a secondary increase in baclofen concentrations into the therapeutic range after an initial decrease, probably due to its slow release from the central nervous system and lipid stores. $^{5}$ This may explain the delay in presentation of our patient.

Convulsions after withdrawal of baclofen are well reported in adults. ${ }^{1-4}$ Convulsions after withdrawal of exposure to baclofen during intrauterine life have not been reported; this is the first such report to the Committee on Safety of Medicines.

Competing interests: None declared.

Barker I, Grant IS. Convulsions after abrupt withdrawal of baclofen Lancet 1982;2:556-7.

2 Hyser CL, Drake ME. Status epilepticus after baclofen withdrawal. J Nat Med Assoc 1984;76:533-8.

3 Kofler M, Lewis AA. Prolonged seizure activity after baclofen withdrawal. Neurology 1992;42:697-8.

4 Terence CF, Formm GH. Complications of baclofen withdrawal. Arch Neurol 1981:38:588-9.

5 Lipscomb DJ, Meredith TJ. Baclofen overdose. Postgrad Med J 1980;56:108-9

\section{Endpiece}

\section{Pleasures}

The three most dangerous are the pleasures of the table, the hunting after honours, and the possession of riches. These desires increase with the age of men.

J Cornaro (1467-1566) Discorsi della vita sobra, Venice, 1620 . Translated by W Jones, Some methods of obtaining a long and healthy life. Edinburgh: A Donaldson, 1768:59-60.

Submitted by Jeremy Hugh Baron,

honorary professorial lecturer, New York 